\title{
A General Framework for Multiple Vehicle Time-Coordinated Path Following Control
}

\author{
Reza Ghabcheloo, Isaac Kaminer, A. Pedro Aguiar, Antonio Pascoal
}

\begin{abstract}
This paper describes a general framework for the study of multiple vehicle, time-coordinated path following (TCPF) control problems. An example is the situation where a group of vehicles is tasked to maneuver and arrive at preassigned final positions at the same time in a collision-free manner, while reducing some optimality criterion. The time of arrival is not fixed a priori, and the vehicles must negotiate their speeds along the spatial paths that they follow in order to arrive simultaneously and avoid collision. The general framework adopted leads to integrated solutions to TC-PF problems that unfold in three steps: 1) Generation of Deconflicted Trajectories for a group of vehicles, 2) Path Following for each vehicle along its assigned path, and 3) Coordination of the relative motion of the vehicles along their paths, so as to guarantee deconfliction and meet desired temporal constraints such as equal times of arrival. The last step is accomplished by varying the speed of each vehicle about the nominal speed profile computed in step 1 , based on the exchange of information with its neighbors. The paper formulates the problem mathematically, offers a general framework for its solution, and illustrates the efficacy of the proposed methodology in simulation with dynamic models of Autonomous Underwater Vehicles (AUVs).
\end{abstract}

\section{INTRODUCTION}

This paper introduces a general framework for the study of a class of multiple vehicle control problems that will henceforth be referred to as Time-Coordinated Path Following (TC$\mathrm{PF})$. The motivation for this work stems from the practical need to develop controllers enabling a group of vehicles to maneuver cooperatively under tight spatial, temporal, and energy constraints.

As an application example, consider the scenario where multiple autonomous marine vehicles - that have been launched and are scattered in the ocean - must execute a cooperative mission underwater, adopting a desired geometrical formation [10]. To this effect, and while still at the surface, the vehicles must maneuver from their initial positions and reach formation at a desired speed, at the diving site. Only then can the underwater mission segment start. Because the vehicles may be operating in a restricted area and in the vicinity of support ships, the initial go-to-formation maneuver must be executed in such a way as to avoid collisions (that is, in a deconflicted manner). Furthermore,

Research supported in part by projects GREX / CEC-IST (Contract No. 035223), NAV-Control / FCT-PT (PTDC/EEA-ACR/65996/2006), and Co3-AUVs (EU FP7 under grant agreement n. 231378), the FCT-ISR/IST plurianual funding program, the FREESUBNET RTN of the CEC, NSF Grant ECS-0242798, and the Academy of Finland.

Reza Ghabcheloo is with the Department of Inteligent Hydraulics and Automation, Tampere Univ of Technology, P.O.Box 589, 33101 Tampere, Finland reza.ghabcheloo@tut.fi

Isaac Kaminer is with the Department of Mechanical and Astronautical Engineering, Naval Postgraduate School, Monterey, CA 93943, USA kaminer anps. navy . edu

A. Pedro Aguiar and Antonio Pascoal are with the Dept. Electrica Engineering and Computers and the Institute for Systems and Robotics, Instituto Superior Técnico, Av. Rovisco Pais, 1, 1049-001 Lisboa, Portugal \{pedro, antonio\}@isr.ist.utl.pt the vehicles must arrive at their target positions at the same time. This procedure is in striking contrast with the rather unpractical attempt to make each vehicle go independently to its target position and loiter there, a task that would be hard to do in the presence of current disturbances and minimum speed requirements for adequate control authority. Another example is the case where the times of arrival of the vehicles at their targets must be separated by specified clearance intervals. Related application examples are also important in the areas of space, air, and land robotics.

The TC-PF control problem incorporates in its formulation strict spatial and temporal constraints. Therefore, its solution requires that a number of tools be brought together to deal with trajectory planning, path following, and cooperative vehicle control in an integrated manner. To better appreciate the scope of the problem at hand, the reader is referred to relevant work on multiple vehicle trajectory planning available in the literature. See for example [16], [18] where the authors describe how a set of trajectories are generated such that a number of vehicles will maneuver and arrive at their final destinations without collision, should they follow the assigned trajectories perfectly. Clearly, the need arises to adopt other strategies for the problem at hand Notice also that trajectory planning algorithms are mainly centralized, computationally demanding, and require high communication bandwidth among the vehicles for proper multiple vehicle trajectory tracking, that is, to accurately track curves that are defined in space and parameterized by time, explicitly. In fact, should one of the vehicles deviate considerably from its planned spatial and temporal schedule (due to environmental disturbances or temporary failures), replanning becomes necessary. Multiple vehicle control schemes that rely on open-loop,"pure planning" strategies of this type have another weak point because they rely on trajectory tracking and, as is well known, the latter has performance limitations that cannot possibly be overcome by any controller structure [2].

To deal with the above problems, the present paper relies on path following rather than trajectory tracking techniques. By exploiting this central idea, a general framework is developed that yields an efficient solution to TC-PF problems. The solution proposed consists of three steps: First, extending the methods exposed in [12], deconflicted trajectories are generated for a group of vehicles. At the end of this step, the trajectories obtained are conveniently re-parametrized by a variable that we call virtual time, leading to a set of spatial paths, together with the corresponding nominal vehicle speed profiles along them. The second step involves the design of path following algorithms to steer each vehicle along its assigned path, while tracking the corresponding speed profile. Here, absolute time does not play any role. Finally, the last step aims to coordinate the relative motion of the 


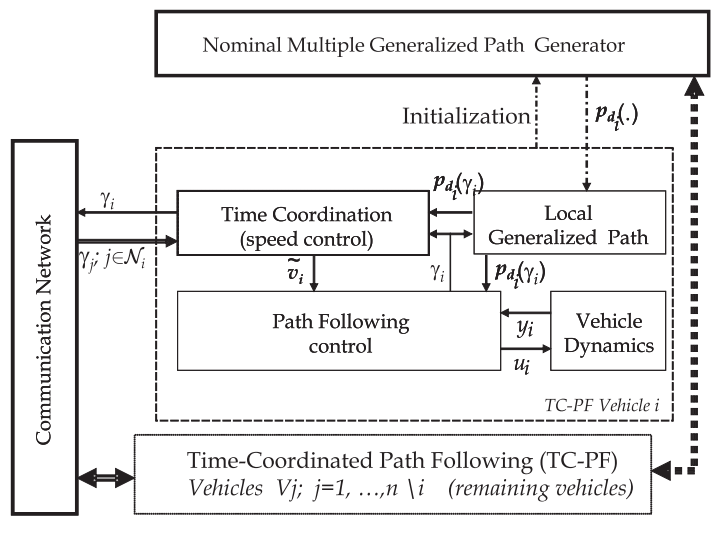

Fig. 1. Architecture of Time-Coordinated Path Following

vehicles along their paths, so as to guarantee deconfliction and meet desired temporal constraints such as equal times of arrival. This is done by varying the speed of each vehicle about the nominal speed profile computed in the first step, based on the exchange of information with its neighbors. The information exchanged is related to the virtual time referred to above. The resulting scheme lends itself to a rigorous formulation and avoids replanning except for the situation where, due to strong disturbances, the vehicles deviate considerably from the paths or fail to meet required temporal constraints.

The mathematical set-up that we adopt is sufficiently general to allow for the consideration of very large classes of algorithms for trajectory generation, path following, and coordinated-path following. For background material on these topics, the reader is referred to [22], [12], [9], [20], and [6], [7], respectively.

Notation. $|\cdot|$ denotes the standard Euclidean norm of a vector in $\mathbb{R}^{n},\|u\|_{I}$ is the (essential) supremum norm of a signal $u:[0, \infty) \rightarrow \mathbb{R}^{n}$ on an interval $I \subset[0, \infty)$, and $f \circ g()=.f(g()$.$) is the composition of functions. In what$ follows, $\mathcal{I}=\{1, \ldots, n\}, p_{d_{i}}^{\gamma_{i}}=\frac{\partial p_{d_{i}}}{\partial \gamma_{i}}$, and $a \oplus b=\max \{a, b\}$.

\section{Time-Coordinated Path Following}

This section introduces the TC-PF control architecture for a group of $n$ dynamically decoupled vehicles represented by general systems of the form

$$
\dot{x}_{i}=f_{i}\left(x_{i}, u_{i}, \omega_{i}\right), y_{i}=g_{i}\left(x_{i}, \nu_{i}\right), p_{i}=h_{i}\left(x_{i}\right),
$$

where $x_{i} \in \mathbb{R}^{n_{i}}$ denotes the state of vehicle $i, u_{i} \in \mathbb{R}^{m_{i}}$ its control input, $y_{i} \in \mathbb{R}^{p_{i}}$ its measured noisy output, $\omega_{i}$ an input disturbance, and $\nu_{i}$ measurement noise. The output $p_{i} \in \mathbb{R}^{q_{i}}$ is the position of vehicle $i$ in an Inertial frame.

The methodology proposed for TC-PF control unfolds in three basic steps that correspond to the three subsystems in Figure 1, described as follows:

- Deconflicted generalized path generation. Feasible time trajectories $p_{d_{i}}(t) ; i \in \mathcal{I}$ are generated that guarantee deconfliction in time with spatial clearance $\bar{E}>0$, that is $\left\|p_{d_{i}}(t)-p_{d_{j}}(t)\right\|>\bar{E}, \quad \forall t>0, \forall i, j \in \mathcal{I}, i \neq j$, and simultaneous arrival of the vehicles at their final destinations. Having generated the desired trajectories parameterized by a single variable $t$, for each vehicle $i \in \mathcal{I}$ we define a desired feasible spatial path as $p_{d_{i}}\left(\gamma_{i}\right): \mathbb{R} \rightarrow \mathbb{R}^{q_{i}}$ parameterized by a free variable $\gamma_{i} \in \mathbb{R}$ to be defined later and a generalized path by

$$
\mathbf{p}_{\mathbf{d}_{\mathbf{i}}}\left(\gamma_{i}\right):=\operatorname{col}\left(p_{d_{i}}\left(\gamma_{i}\right), p_{d_{i}}^{\gamma_{i}}\left(\gamma_{i}\right)\right) \text {. }
$$

A generalized path includes the spatial path itself as well the nominal speed profile of the vehicle along it.

- Path-following. The path following subsystem $i$ is a dynamical system whose inputs are the desired generalized path $\mathbf{p}_{\mathbf{d}_{\mathbf{i}}}\left(\gamma_{i}\right)$, a correction speed $\tilde{v}_{d_{i}}$ signal from the timecoordination system described next, and local measurements $y_{i}$. Its outputs are the variable $\gamma_{i}$ and the vehicle's control signal $u_{i}$, computed so as to make $p_{i}$ reach and follow $p_{d_{i}}$ with the assigned speed. Notice that the dynamics of the parameterizing variable $\gamma_{i}$ are defined internally at this stage and play the role of an extra design knob to tune the performance of the path following control law.

- Time Coordination. A dynamical system whose inputs are local measurement $y_{i}$, the desired generalized path $\mathbf{p}_{\mathbf{d}_{\mathbf{i}}}$, the path-variables $\gamma_{i}$ and $\gamma_{j} ; j \in \mathcal{N}_{i}$, where $\mathcal{N}_{i}$ denotes the set of vehicles that vehicle $i$ communicates with. Its output is the correction speed signal $\tilde{v}_{d_{i}}$ which is used to synchronize vehicle $i$ with its neighbors.

\section{A. Deconflicted Generalized Path Generation}

A typical trajectory generation problem aimed at obtaining equal times of arrival can be stated as follows. Consider a fleet of $n$ vehicles described by (1). For each vehicle, compute a feasible trajectory, satisfying a given optimality criterion and extending from a known initial position $P_{0_{i}}$ to a given final position $P_{f_{i}}$, such that all the trajectories are deconflicted in time, end at the same time, and satisfy a given number of constraints ${ }^{1}$. Should the problem have a solution, the trajectory planning algorithm produces a nominal trajectory $p_{d_{i}}\left(t_{p}\right)$ to be followed by vehicle $i$, parameterized by $t_{p} \in \mathbb{T}_{f}:=\left[0, t_{f}\right]$ where $t_{f}$ denotes the length of the maneuver. For the sake of clarity, we refer to the time parameter $t_{p}$ as virtual-time. This is done to distinguish it from the real time $t$ that unfolds during the execution of a mission. We now make the key observation that virtualtime can be simply viewed as a variable that parameterizes the spatial paths derived from the trajectories above and can therefore be identified with the path parameters $\gamma_{i} ; i \in \mathcal{I}$ introduced before. These paths, together with the resulting vehicle speed assignments (specified as functions of $\gamma_{i}$ ), are all that is required for path following, which will dictate how $\gamma_{i}$ actually evolves in time.

Assumption 1. We assume the functions $p_{d_{i}}($.$) are Lips-$ chits on $\mathbb{T}_{f}$ with constant $L$, that is,

$$
\left\|p_{d_{i}}\left(t_{a}\right)-p_{d_{i}}\left(t_{b}\right)\right\|<L\left|t_{a}-t_{b}\right| ; \forall i \in \mathcal{I},
$$

and that the trajectories are deconflicted in time to satisfy

$$
\left\|p_{d_{i}}\left(t_{a}\right)-p_{d_{j}}\left(t_{a}\right)\right\|>\bar{E} ; \forall t_{a} \in \mathbb{T}_{f}, \forall i, j \in \mathcal{I} ; i \neq j .
$$

Lemma 1: Let $p_{d_{i}}($.$) satisfy Assumption 1$ and define $\delta=$ $(\bar{E}-E) / L$ for arbitrarily $E<\bar{E}$. If $\left|t_{a}-t_{b}\right| \leq \delta$, then

$$
\left\|p_{d_{i}}\left(t_{a}\right)-p_{d_{j}}\left(t_{b}\right)\right\|>E, \quad \forall i, j \in \mathcal{I} ; i \neq j, \forall t_{a}, t_{b} \in \mathbb{T}_{f} .
$$

Lemma 1 can be interpreted as follows: to guarantee the minimum spatial clearance of $E$, the mismatch $\left|t_{a}-t_{b}\right|$ of virtual-time variables must not exceed $\delta$.

\footnotetext{
${ }^{1}$ Examples include the physical limitations of the vehicles, often described as acceleration constraints. The initial and final desired speeds, as well as regions to be avoided, are also considered as constraints.
} 
Definition 1: We denote by $\mathcal{Z}_{d}$ the set of $n$-tuple $\mathbf{p}_{\mathbf{d}}=$ $\left(\mathbf{p}_{\mathbf{d}_{\mathbf{1}}}(),. \ldots, \mathbf{p}_{\mathbf{d}_{\mathbf{n}}}().\right)$, where $\mathbf{p}_{\mathbf{d}_{\mathbf{i}}}$ is defined by (2) and $p_{d_{i}}\left(t_{p}\right)$ are feasible trajectories that satisfy Assumption 1, together with the initial and final constraints $p_{d_{i}}(0)=P_{0_{i}}$ and $p_{d_{i}}\left(t_{f}\right)=P_{f_{i}}$, respectively for all $i \in \mathcal{I}$, where $t_{f}$ denote the simultaneous time of arrival.

\section{B. Path Following}

Definition 2: (see [1]) Consider a set of $n$ vehicles with dynamics (1). We say that a controller $\Sigma_{P F i} ; i \in \mathcal{I}$ given by

$$
\begin{aligned}
\Sigma_{P F i}: \dot{x}_{P F i} & =\mathcal{F}_{P F i}\left(x_{P F i}, y_{i}, \mathbf{p}_{\mathbf{d}_{\mathbf{i}}}, \tilde{v}_{d_{i}}\right), \\
u_{i} & =\mathcal{H}_{P F i}\left(x_{P F i}, y_{i}, \mathbf{p}_{\mathbf{d}_{\mathbf{i}}}, \tilde{v}_{d_{i}}\right)
\end{aligned}
$$

solves robustly the output path-following problem if, for every generalized path $\mathbf{p}_{\mathbf{d}_{\mathbf{i}}}$ taken from $\mathcal{Z}_{d}$, there exist an error signal $\mathbf{e}_{\mathbf{i}}$ and functions $\sigma_{\omega}^{e}, \sigma_{\nu}^{e}, \sigma^{e}, \sigma_{\tilde{v}_{d_{i}}}^{e} \in \mathcal{K}_{\infty}$, such that for bounded signals $\omega_{i}, \nu_{i}$, and $\tilde{v}_{d_{i}}$, all the states of the closed-loop system (1) and (5) with the exception of $\gamma_{i}(t)$ are bounded, the path-following errors $e_{i}(t):=p_{i}(t)-p_{d_{i}}\left(\gamma_{i}(t)\right)$ and the speed errors $e_{\dot{\gamma}_{i}}(t):=\dot{\gamma}_{i}(t)-1, \forall i \in \mathcal{I}$ satisfy the detectability condition

$$
\left|e_{i}(t)\right| \oplus\left|e_{\dot{\gamma}_{i}}(t)\right| \leq \sigma^{e}\left(\left\|\mathbf{e}_{\mathbf{i}}\right\|_{[0, t]}\right), \quad \forall i \in \mathcal{I}
$$

and $\mathbf{e}_{\mathbf{i}}$ is input-to-output stable (IOS) with respect to $\omega_{i}, \nu_{i}$, and $\tilde{v}_{d_{i}}$, that is,

$$
\left|\mathbf{e}_{\mathbf{i}}(t)\right| \leq \sigma_{\omega}^{e}\left(\left\|\omega_{i}\right\|_{[0, t]}\right) \oplus \sigma_{\nu}^{e}\left(\left\|\nu_{i}\right\|_{[0, t]}\right) \oplus \sigma_{\tilde{v}_{d_{i}}}^{e}\left(\left\|\tilde{v}_{d_{i}}\right\|_{[0, t]}\right) .
$$

For detailed defintions of ISS and IOS, see [11], [21]. In Section III-B we give the example of a path following controller for a fully actuated AUV subjected to water current disturbances.

\section{Time Coordination}

Let the position of vehicle $i$ at time $t$ be given by $p_{d_{i}}\left(\gamma_{i}(t)\right)$, where $\gamma_{i}$ is a variable that parametrizes the path $p_{d_{i}}($.$) . The trajectory generation at Step 1$ guarantees the following two facts: 1) if $\forall t>0, \gamma_{i}(t)=\gamma_{j}(t)$, then vehicle $i$ and vehicle $j$ will remain deconflicted; 2 ) vehicle $i$ will follow its trajectory as planned if $\gamma_{i}(t)=t$ and the initial time is set to 0 . In the non ideal case where due to disturbances the vehicles deviate from the planned trajectories, one can guarantee that the vehicles will be at least $E$ meters apart whenever $\left|\gamma_{i}(t)-\gamma_{j}(t)\right|<\delta$ for all $t>0$ and all $i, j \in \mathcal{I}$.

Using the above observations, we seek closed-loop dynamics for $\dot{\gamma}_{i}$ to achieve the following two objectives:

1) The errors $\left|\gamma_{i}-\gamma_{j}\right|$ must remain sufficiently small to guarantee quasi-simultaneous time of arrival, and to ensure collision-free maneuvers. In addition, $\left|\gamma_{i}-\gamma_{j}\right|$ must converge to zero in a disturbance-free situation.

2) In the absence of disturbances, the dynamics of $\gamma_{i}$ must verify $\dot{\gamma}_{i}=1$, so as to recover the planned trajectories and optimality is retained. Notice that the mission will be "near optimal" in the presence of disturbances.

To meet the objectives above, for each vehicle the nominal speed profile is perturbed by a corrective speed $\tilde{v}_{d_{i}}$ that is function of the errors $\left|\gamma_{i}-\gamma_{j}\right|$. These adjustments are done by exchanging coordination information (virtual-time variables $\gamma_{j}$ ) among vehicles using the supporting communication network. The problem of synchronizing $\left|\gamma_{i}-\gamma_{j}\right|$ has close affinity to agreement problems, consensus algorithms, and multi-agent cooperation. See [4], [14], [17] and the references therein. The tools required to study these problems are diverse and include graph theory [3], [19]. In practice, some assumptions must me made with respect to the connectivity of the underlying communication graph to ensure adequate behaviour of the synchronization system. Another issue of considerable importance is the impact of the rate of communications on the convergence rates of appropriately defined error variables. In this paper, we will not examine this issue in detail. See [7], [4], [1] and the references therein for details.

Definition 3: Consider a set of $n$ vehicles with dynamics (1) and a generalized path $\mathbf{p}_{\mathbf{d}} \in \mathcal{Z}_{d}$. We assume the vehicles exchange information over a communication network with sufficient rate, the graph of which is supposed to satisfy certain connectivity properties. In particular, vehicle $i$ receives variables $\gamma_{j}\left(t_{k}\right)$ from vehicles $j \in \mathcal{N}_{i}$, where $\mathcal{N}_{i}$ denotes the set of neighboring vehicles of vehicle $i$ at time $t_{k}$. Let $\tilde{\gamma}_{i}=\left[\gamma_{i}-\gamma_{j}\right]_{j \in \mathcal{N}_{i}}, \bar{\xi}=\operatorname{col}\left(\xi, \tilde{v}_{d}\right)$, and $\tilde{v}_{d}:=\left[\tilde{v}_{d_{i}}\right]_{i \in \mathcal{I}}$. We say that a set of time-coordination controllers $\Sigma_{C C i}, i \in \mathcal{I}$

$\Sigma_{C C i}: \dot{x}_{C C i}=\mathcal{F}_{C C i}\left(x_{C C i}, y_{i}, \mathbf{p}_{\mathbf{d}_{\mathbf{i}}}, \tilde{\gamma}_{i}\left(t_{k}\right)\right)$,

$$
\tilde{v}_{d_{i}}=\mathcal{H}_{C C i}\left(x_{C C i}, y_{i}, \mathbf{p}_{\mathbf{d}_{\mathbf{i}}}, \tilde{\gamma}_{i}\left(t_{k}\right)\right) ; t_{k} \leq t<t_{k+1}
$$

solves robustly the time-coordination problem if there exist functions $\sigma^{\xi}, \sigma_{\nu}^{\xi}, \sigma_{\gamma}^{\xi}, \sigma_{e}^{\xi} \in \mathcal{K}_{\infty}$, constant $\epsilon>0$, and a coordination error signal $\xi$ that satisfy the detectability property

$$
\max _{i \in \mathcal{I} ; j \in \mathcal{N}_{i}}\left|\gamma_{i}(t)-\gamma_{j}(t)\right| \leq \sigma^{\xi}\left(\|\xi\|_{[0, t)}\right)<\delta,
$$

where $\delta$ is defined in Lemma 1, and the input-to-output practical stability property

$$
\|\bar{\xi}\| \leq \sigma_{\nu}^{\xi}\left(\|\nu\|_{[0, t]}\right) \oplus \sigma_{e}^{\xi}\left(\|\mathbf{e}\|_{[0, t)}\right) \oplus \epsilon,
$$

where $\nu:=\left[\nu_{i}\right]_{i \in \mathcal{I}}$, and $\mathbf{e}:=\left[\mathbf{e}_{\mathbf{i}}\right]_{i \in \mathcal{I}}$.

\section{Time-Coordinated Path Following}

We now address formally the TC-PF control problem.

Theorem 1: Consider the closed-loop system $\Sigma_{C L}$ consisting of $n$ vehicles with dynamics (1) and feedback control system (5) and (8). Suppose that each path following controller $\Sigma_{P F i}$ and coordinated controller $\Sigma_{C C i}$ solve robustly the output path-following problem and the time-coordination problem, respectively, that is, inequalities (6)-(7), (9)-(10) hold. Suppose further that

$$
\sigma_{\tilde{v}_{d}}^{e} \circ \sigma_{e}^{\xi}(r)<r, \quad \forall r>r_{0} .
$$

Then, the overall closed-loop system solves robustly the TCPF problem, that is, there exist functions $\sigma^{\bar{e}}, \sigma_{\omega}^{\bar{e}}, \sigma_{\nu}^{\bar{e}} \in \mathcal{K}_{\infty}$, a positive number $\epsilon$, and a signal error $\overline{\mathbf{e}}$ such that for bounded signals $\omega:=\left[\omega_{i}\right]_{i \in \mathcal{I}}$ and $\nu:=\left[\nu_{i}\right]_{i \in \mathcal{I}}$, all the states of the closed-loop system $\Sigma_{C L}$ with the exception of $\gamma(t):=\left[\gamma_{i}\right]_{i \in \mathcal{I}}$ are bounded, the path-following errors, speed errors, and coordination errors satisfy the detectability condition

$$
\max _{i \in \mathcal{I}}\left(\left|e_{i}(t)\right| \oplus\left|e_{\dot{\gamma}_{i}}(t)\right| \oplus \max _{j \in \mathcal{N}_{i}}\left(\gamma_{i}-\gamma_{j}\right)\right) \leq \sigma^{\bar{e}}\left(\|\overline{\mathbf{e}}\|_{[0, t]}\right)
$$

and $\overline{\mathbf{e}}$ is input-to-output stable with respect to $\omega$ and $\nu$, i.e.,

$$
|\overline{\mathbf{e}}(t)| \leq \sigma_{\omega}^{\bar{e}}\left(\|\omega\|_{[0, t]}\right) \oplus \sigma_{\nu}^{\bar{e}}\left(\|\nu\|_{[0, t]}\right) \oplus \epsilon .
$$

Proof is omitted. See [11] for an applicaiton of the small-gain theorem. 


\section{ILLUSTRATIVE EXAMPLE: TC-PF OF MULTIPLE AUVS}

In this section, we provide a specific solution to the subproblems discussed before. For simplicity of exposition, we adopt a simple 2D kinematic model for fully actuated AUVs where the inputs are linear velocity and angular rate [13], [5].

\section{A. Generation of suboptimal deconflicted trajectories}

This section describes a solution to the problem of computing 2D deconflicted trajectories for multiple AUVs that satisfy the properties in Definition 1.

We denote by $p_{r_{i}}\left(\tau_{i}\right)=\left(x_{1_{i}}\left(\tau_{i}\right), x_{2_{i}}\left(\tau_{i}\right)\right)^{T}$ a desired path to be followed by AUV $i$, parameterized by the virtual arc $\tau_{i} \in\left[0, \tau_{f_{i}}\right]$, where $\tau_{f_{i}}$ is the total virtual arc length between $P_{0_{i}}$ and $P_{f_{i}}$. Following [22], we let the coordinates $x_{1}$ and $x_{2}$ be represented by algebraic polynomials of degree $N$ of the form

$$
x_{k}(\tau)=\sum_{i=0}^{N} a_{k, i} \tau^{i} ; k=1,2,
$$

where, for ease of notation, we have dropped the vehicle index. The degree $N$ of the polynomials $x_{k}(\tau) ; k=1,2$ is determined by the number of boundary conditions that must be satisfied. In the sequel, we will let the prime sign / stand for $\partial / \partial \tau$ and /I for the second derivative operator. Further, dot signs $\dot{-}$ and $\ddot{-}$ denote the first and the second time derivatives. Given $\tau_{f}$ and terminal constraints $x_{k}(0), x_{k}^{\prime}(0), x_{k}^{\prime \prime}(0), x_{k}\left(\tau_{f}\right), x_{k}^{\prime}\left(\tau_{f}\right)$, and $x_{k}^{\prime \prime}\left(\tau_{f}\right)$, for $k=$ 1,2 , the coefficients of $N=5$ th order polynomial are easily computed from

$$
\left(\begin{array}{cccccc}
1 & 0 & 0 & 0 & 0 & 0 \\
0 & 1 & 0 & 0 & 0 & 0 \\
0 & 0 & 2 & 0 & 0 & 0 \\
1 & \tau_{f} & \tau_{f}^{2} & \tau_{f}^{3} & \tau_{f}^{4} & \tau_{f}^{5} \\
0 & 1 & 2 \tau_{f} & 3 \tau_{f}^{2} & 4 \tau_{f}^{3} & 5 \tau_{f}^{4} \\
0 & 0 & 2 & 6 \tau_{f} & 12 \tau_{f}^{2} & 20 \tau_{f}^{3}
\end{array}\right)\left(\begin{array}{c}
a_{k, 0} \\
a_{k, 1} \\
a_{k, 2} \\
a_{k, 3} \\
a_{k, 4} \\
a_{k, 5}
\end{array}\right)=\left(\begin{array}{c}
x_{k}(0) \\
x_{k}^{\prime}(0) \\
x_{k}^{\prime \prime}(0) \\
x_{k}\left(\tau_{f}\right) \\
x_{k}^{\prime}\left(\tau_{f}\right) \\
x_{k}^{\prime \prime}\left(\tau_{f}\right)
\end{array}\right) .
$$

It is important to notice that the parameterization (14) completely determines the AUV's spatial profile, that is, a 2D path that satisfies all boundary conditions by construction. It now remains to address the time related requirements which include deconfliction in time and simultaneous arrival. To deal with this situation and avoid collisions, we need to label each point on the path $i$ with a time tag. This is equivalent to defining $\dot{\tau}_{i}$ or, equivalently, defining speed profiles of the AUV $i ; i \in \mathcal{I}$ along the paths, since $\dot{p}_{r_{i}}=\dot{\tau}_{i} p_{r_{i}}^{\prime}$. We define $\eta_{i}\left(\tau_{i}\right)=\frac{d \tau_{i}}{d t}$. With the above definitions, the temporal and spatial derivatives of $p_{r_{i}}$ satisfy

$$
\begin{aligned}
& \dot{p}_{r_{i}}=\eta_{i} p_{r_{i}}^{\prime} \\
& \ddot{p}_{r_{i}}=\eta_{i} \eta_{i}^{\prime} p_{r_{i}}^{\prime}+\eta_{i}^{2} p_{r_{i}}^{\prime \prime} .
\end{aligned}
$$

For the purposes of this paper it is sufficient to choose $\eta_{i}$ to be an affine function of $\tau_{i}$, that is, $\eta_{i}\left(\tau_{i}\right)=\eta_{0_{i}}+\frac{\eta_{f_{i}}-\eta_{0_{i}}}{\tau_{f_{i}}} \tau_{i}$ with $\eta_{0_{i}}=\left\|\dot{p}_{r_{i}}(0)\right\|$, and $\eta_{f_{i}}=\left\|\dot{p}_{r_{i}}\left(t_{f}\right)\right\|$, where $t_{f}$ is selected as an optimization parameter. Let $v_{\min }, v_{\max }$ and $a_{\max }$ be predefined bounds on the vehicle's velocity $\left\|\dot{p}_{r_{i}}\right\|$ and acceleration $\left\|\ddot{p}_{r_{i}}\right\|$. By integrating $\dot{\tau}_{i}=\eta_{i}\left(\tau_{i}\right)$, the virtual $\operatorname{arc} \tau_{i}$ and time $t$ are related through the following equations

$$
\tau_{f_{i}}= \begin{cases}\eta_{0_{i}} t_{f}, & \eta_{f_{i}}=\eta_{0_{i}} \\ \frac{\eta_{f_{i}}-\eta_{0_{i}}}{\ln \left(\eta_{f_{i}} / \eta_{0_{i}}\right)} t_{f} & \eta_{f_{i}} \neq \eta_{0_{i}}\end{cases}
$$

$$
\frac{\tau_{i}}{\tau_{f_{i}}}= \begin{cases}\frac{t}{t_{f}}, & \eta_{f_{i}}=\eta_{0_{i}} \\ \frac{\eta_{0_{i}}}{\eta_{f_{i}}-\eta_{0_{i}}}\left(\left(\frac{\eta_{f_{i}}}{\eta_{0_{i}}}\right)^{\frac{t}{t_{f}}}-1\right) . & \eta_{f_{i}} \neq \eta_{0_{i}}\end{cases}
$$

To formally introduce deconfliction in time constraints, we define the time stamped trajectory $p_{d_{i}}(t):=p_{r_{i}}\left(\tau_{i}(t)\right)$, where $\tau_{i}(t)$ is given by (18). Having fixed the final time $t_{f}$, the total arc lengths $\tau_{f_{i}}$ are computed using (17) and the spatial paths $p_{r_{i}}\left(\tau_{i}\right)$ and speed profiles $\dot{p}_{r_{i}}$ are given by (15) and (16), respectively. We employ a direct method of calculus of variation to determine the optimal simultaneous arrival time $t_{f}$. We assume the optimal arrival time $t_{f}$ must lie inside a pre-defined interval $\left[t_{1}, t_{2}\right]$. The mathematical problem of interest can now be stated formally as that of computing

$$
t_{f}^{o p t}=\arg \min _{t_{f} \in\left[t_{1}, t_{2}\right]} \sum_{i=1}^{n} w_{i} J_{i}
$$

subject to (4) for deconfliction in time, and

$$
\begin{gathered}
v_{\min } \leq \eta_{i}\left(\tau_{i}\right)\left\|p_{r_{i}}^{\prime}\left(\tau_{i}\right)\right\| \leq v_{\max } \\
\eta_{i}\left(\tau_{i}\right)\left\|\eta_{i}^{\prime}\left(\tau_{i}\right) p_{r_{i}}^{\prime}\left(\tau_{i}\right)+\eta_{i}\left(\tau_{i}\right) p_{r_{i}}^{\prime \prime}\left(\tau_{i}\right)\right\| \leq a_{\max }
\end{gathered}
$$

$\forall \tau_{i} \in\left[0, \tau_{f_{i}}\right]$, where $w_{i} ; i=1, \ldots, n$ are positive weights. The criterion $J_{i}$ may be taken as the total energy consumption along a trajectory. We let $\int_{0}^{\tau_{f_{i}}}\left\|p_{r_{i}}^{\prime}(\tau)\right\|^{3} \eta_{i}(\tau)^{3} d \tau$, in the examples presented here. The rationale for this cost function stems from the fact that the instantaneous power required for vehicle maneuvering is proportional to the cube of speed. This constrained optimization problem over a single optimization parameter can be solved in real-time using any zero-order optimization technique (see [22]).

Notice that a Lipschits constant for functions $p_{d_{i}}($.$) can be$ computed as $L=v_{\max }=\max _{t \in\left[0, t_{f}\right]} \dot{p}_{d_{i}}(t)$. The outcome of this step is a set of trajectories $p_{d_{i}}($.$) from which an n$ tuple generalized path $\mathbf{p}_{\mathbf{d}} \in \mathcal{Z}_{d}$ is generated.

\section{B. Path following: single AUV}

Consider an AUV depicted in Figure 2, together with a spatial path $\Gamma$ parameterized by $p_{d_{i}}\left(\gamma_{i}\right)$ obtained using the method described in Section III-A. In the figure, $P$ is an arbitrary point on the path to be followed and $Q$ is the center of the mass of the vehicle. Associated with $P$, consider the Serret-Frenet $\{\mathrm{T}\}$. The center of mass $Q$ can be expressed either as $p_{i}$ in the inertial reference frame $\{\mathrm{U}\}$, or as $\left(x_{e}, y_{e}\right)$ in $\{\mathrm{T}\}$. Define two frames with their origin at the center of mass of the vehicle: i) the body-fixed frame denoted $\{\mathrm{B}\}$ with its $x$-axis along the main axis of the body, and ii) the flow frame denoted $\{\mathrm{F}\}$ with its $x$-axis along the vehicle total velocity whose magnitude is denoted by $v_{i}$. Further let $\psi_{T}$ and $\psi_{F}$ denote the angle from $\{\mathrm{T}\}$ to $\{\mathrm{U}\}$ and from $\{\mathrm{F}\}$ to $\{\mathrm{U}\}$, respectively. The yaw angle of the vehicle will be denoted $\psi_{B}$, and $r_{i}=\dot{\psi}_{B}$ is the angular speed of the vehicle. We will consider water currents as disturbances acting on the vehicle. We let $v_{c}$ and $\psi_{c}$ denote the speed and orientation, respectively, of the water current in the inertial frame. The desired speed profile is given by $v_{d_{i}}\left(\gamma_{i}\right)=\left\|p_{d_{i}}^{\gamma_{i}}\left(\gamma_{i}\right)\right\|$, and $\dot{s}_{i}=v_{d_{i}} \dot{\gamma}_{i}$ where $s_{i}$ is the signed curvilinear abscissa of $P$ along the path. Simple computations lead to the kinematics of the vehicle in the error coordinates $\left(x_{e_{i}}, y_{e_{i}}, \psi_{e_{i}}\right)$ as

$$
\left\{\begin{array}{l}
\dot{x}_{e_{i}}=\left(y_{e_{i}} c_{c_{i}}-1\right) v_{d_{i}} \dot{\gamma}_{i}+v_{i} \cos \psi_{e_{i}}+v_{c} \cos \psi_{c T} \\
\dot{y}_{e_{i}}=-x_{e_{i}} c_{c_{i}} v_{d_{i}} \dot{\gamma}_{i}+v_{i} \sin \psi_{e_{i}}+v_{c} \sin \psi_{c T} \\
\dot{\psi}_{e_{i}}=r_{i}-c_{c_{i}} v_{d_{i}} \dot{\gamma}_{i}+\dot{\beta}_{i}
\end{array}\right.
$$




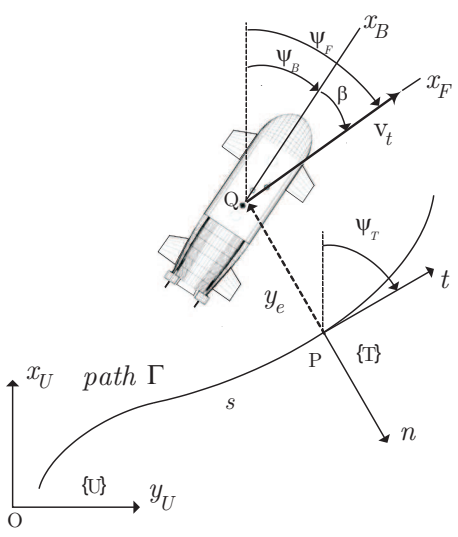

Fig. 2. Frames and error variables

where $\psi_{e_{i}}=\psi_{F}-\psi_{T}, \psi_{c T}=\psi_{c}-\psi_{T}, \beta_{i}=\psi_{F}-\psi_{B}$ is the angle of side-slip, and $c_{c_{i}}$ is path curvature at point $P$. See [8] for the details of the derivation. As seen from (21), the equations of motion are driven by $v_{i}, r_{i}$ and term $\dot{\gamma}_{i}$ that plays the role of an extra control signal.

Lemma 2: [Path Following] Consider AUV $i$ with the equations of motion (21) together with the path $\left(p_{d_{i}}, p_{d_{i}}^{\gamma_{i}}\right)$ to be followed. Let

$$
\begin{aligned}
& v_{i}=v_{d_{i}}+\tilde{v}_{d_{i}} \\
& r_{i}=c_{c_{i}} v_{d_{i}} \dot{\gamma}_{i}+\dot{\zeta}_{i}-k_{3}\left(\psi_{e_{i}}-\zeta_{i}\right)-k_{3} y_{e_{i}} v_{i} \sigma_{i}-\dot{\beta}_{i}
\end{aligned}
$$

where $\zeta_{i}=\arcsin \left(\frac{-k_{2} y_{e_{i}}}{\left|y_{e_{i}}\right|+d_{1}}\right), \sigma_{i}=\frac{\sin \psi_{e_{i}}-\sin \zeta_{i}}{\psi_{e_{i}}-\zeta_{i}}$ and let the dynamics of $P$ on the path be governed by the feedback law

$$
\dot{\gamma}_{i}=\left(1+\frac{\tilde{v}_{d_{i}}}{v_{d_{i}}}\right) \cos \psi_{e_{i}}+\frac{k_{1}}{v_{d_{i}}} \operatorname{sat}\left(x_{e_{i}}\right)
$$

where $k_{1}>0,0<k_{2}<1, k_{3}>0$ and $d_{1}>0$. If the speed correction term $\tilde{v}_{d_{i}}$ satisfies lower bound $\tilde{v}_{d_{i}} \geq v_{m}-v_{d_{i}}$ for some $v_{m}>0$, and $v_{c}$ is small enough, then the PF control defined by (22)-(23) solve robustly the output path following problem defined in Section II-B, where the following IOS relations hold,

$$
\begin{aligned}
\left|e_{i}\right| & \leq \sigma_{1}\left(\left\|v_{c}(t)\right\|_{[0, t]}\right) \\
\left|e_{\dot{\gamma}_{i}}\right| & \leq \sigma_{2}\left(\left\|v_{c}(t)\right\|_{[0, t]}\right) \oplus \sigma_{3}\left(\left\|\tilde{v}_{d_{i}}\right\|_{[0, t]}\right) .
\end{aligned}
$$

for path-following error $e_{i}$ and speed tracking error $e_{\dot{\gamma}_{j}}$. Proof is omitted.

\section{Time-coordinated path following}

The AUVs will adjust their speed according to $\left|\gamma_{i}-\gamma_{j}\right|$ to keep the latter variable small and drive it to zero in the absence of disturbances. This requires a communication network for the exchange of information among AUVs. We avail ourself of some results on graph theory. See [3]. We will assume that the underlying communication graph is UQSC (uniformly quasi-strongly connected). See [15] for the definition of UQSC. We now state the main result of this section.

Theorem 2: [Time-coordinated path-following] Consider $n$ AUVs and $n$-tuple trajectories $\mathbf{p}_{\mathbf{d}} \in \mathcal{Z}_{d}$ generated in Section III-A. Assume each AUV is equipped with the path following algorithm of Lemma 2 and the communication graph is UQSC. For any rate of communication losses and sufficiently small $k_{1} / k_{c}$, the speed correction control law

$$
\tilde{v}_{d_{i}}=\frac{v_{d_{i}}}{\left|\cos \psi_{e_{i}}\right|}\left(1-k_{c} \operatorname{sat}\left(\sum_{j \in \mathcal{N}_{i}} \gamma_{i}-\gamma_{j}\right)\right)-v_{d_{i}}, \forall i \in \mathcal{I}
$$

solves robustly the time-coordinated path following problem defined in Section II-D, with $0<k_{c}<1$. Namely, the speed correction remains bounded, $\tilde{v}_{d_{i}}(t) \geq v_{\min }\left(1-k_{c}\right)-v_{d_{i}}$, and $|\overline{\mathbf{e}}| \leq \sigma_{v_{f}}^{\bar{e}}\left(\left\|v_{c}(t)\right\|_{[0, t]}\right)$ for some $\sigma_{v_{c}}^{\bar{e}} \in \mathcal{K}$, where $\overline{\mathbf{e}}=\max _{i \in \mathcal{I}}\left\{\left|e_{i}\right|,\left|e_{\dot{\gamma}_{i}}\right|\right\} \oplus \xi$ is a TC-PF error and $\xi=$ $\max _{j \in \mathcal{N}_{i}, i \in \mathcal{I}}\left|\gamma_{i}-\gamma_{j}\right|$.

Proof is omitted.

\section{Simulation RESUlts}

As an illustrative example, we consider the problem of deconflicted simultaneous arrival of two AUVs in 2D space.

\section{A. Deconflicted path generation}

Vehicle 1 starts at $x_{1}(0)=(0,0)^{T}[\mathrm{~m}]$ with velocity $v_{1}(0)=(0.5,0)^{T}[\mathrm{~m} / \mathrm{s}]$ and zero acceleration. Vehicle 2 starts at $x_{2}(0)=(0,5)^{T}[\mathrm{~m}]$, with the same velocity and acceleration. The aim is to generate deconflicted trajectories that end at $x_{1}\left(t_{f}\right)=(10,10)^{T}[\mathrm{~m}]$, and $x_{2}\left(t_{f}\right)=$ $(15,10)^{T}[\mathrm{~m}]$ for vehicle 1 and 2 , respectively, for a terminal time $30 \mathrm{~s} \leq t_{f} \leq 60 \mathrm{~s}$. We also require that the final velocities be $(0.75,0)^{T}[\mathrm{~m} / \mathrm{s}]$ and the accelerations be zero. A set of admissible polynomials of order 5 for $t_{f} \in[30,60] \mathrm{s}$ was computed; the optimal simultaneous time of arrival is $t_{f}^{\text {opt }}=$ $46.5 \mathrm{~s}$.

\section{B. Simulations}

The performance of the algorithm developed is illustrated in this section with the help of numerical simulations. The control gains in (22) and (25) are set to $k_{1}=5, k_{2}=1$, $k_{3}=0.34, d_{1}=1$, and $k_{c}=0.5$, in SI units. The first simulation (see Figure 3) was performed for the ideal situation where there are no disturbances. In this case, as planned, both AUVs arrive at their final destinations at $t_{f}=$ $46.8[\mathrm{~s}]$ (close to $t_{f}^{o p t}$ ), and the maneuver is deconflicted. The second set of simulations show the effects of a water current with speed $v_{c}=0.1[\mathrm{~m} / \mathrm{s}]$ and orientation $\psi_{c}=\pi[\mathrm{rad}]$ in the inertial frame. See Figure 4 where the labels along the paths indicate times of arrival of the AUVs at the marked positions. Although it takes longer for the AUVs to arrive at the final destinations, they both arrive at almost the same time $t_{f}=56[\mathrm{~s}]$. Figures 5 and 6 show the time-coordination error and the AUVs distance from each other, respectively, as a function of time. Notice that the coordination error grows in the case of water current, but not so much as to lead to a collision. Figures 5 and 6 include two other tests as well, to further demonstrate the effect of disturbances. When no time-coordination is performed, the spatial clearance goes down to $0.6[\mathrm{~m}]$ and temporal coordination error raises to 6.5 [sec]. When the communication channel is lost $90 \%$ of time, the clearance is still acceptable, $2.9[\mathrm{~m}]$, and the timecoordination errors increase to $3.65[\mathrm{sec}]$.

\section{CONCLUSiON}

The paper developed a general framework for the study of multiple vehicle, time-coordinated path following (TC-PF) problems. With the framework adopted, the solution unfolds in three steps: 1) Generation of deconflicted paths, together with the corresponding nominal vehicle speed profiles along them, 2) Path following for each vehicle along its assigned path, and 3) Coordination of the relative motion of the vehicles along their paths, so as to guarantee deconfliction and equal times of arrival. The paper formulated the problem 


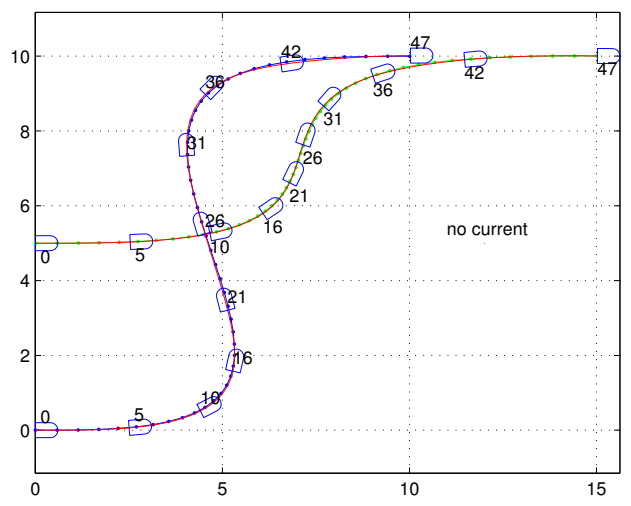

Fig. 3. Desired and actual trajectories of the AUVs; no current

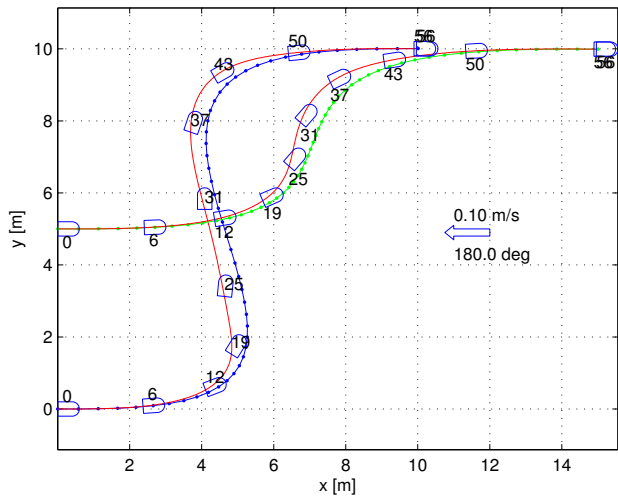

Fig. 4. Desired and actual trajectories of the AUVs; current $v_{c}=0.1[\mathrm{~m} / \mathrm{s}]$

mathematically, offered a general framework for its solution, and illustrated the efficacy of the methodology proposed through simulation with dynamic models of autonomous underwater vehicles. Future work will aim at accommodating logic based communications.

\section{REFERENCES}

[1] Aguiar, A.P., and Pascoal, A. M. (2007), "Coordinated path-following control for nonlinear systems with logic-based communication," Proc. of the 46th IEEE Conf. on Decision and Contrrol, New Orleans, LO.

[2] Aguiar, A.P., Hespanha, J., and Kokotovic, P. (2008), Performance Limitations in Reference-Tracking and Path-Following for Nonlinear Systems, IFAC Automatica, Vol. 44, No. 3, pp. 598-610, Mar. 2008.

[3] Balakrishnan, R. and Ranganathan, K. (2000), A Textbook of Graph Theory, Springer.

[4] Blondel, V. D., Hendrickx, J. M., Olshevsky, A. and Tsitsiklis, J. N. (2005), "Convergence in multiagent coordination, consensus, and flocking," Proc. of the 44th IEEE CDC, Seville, Spain.

[5] Fossen, T.(2002), Marine Control Systems: Guidance, Navigation and Control of Ships, Rigs and Underwater Vehicles, Marine Cybernetics AS.

[6] Ghabcheloo, R., Aguiar, A. P., Pascoal, A., Silvestre, C., Hespanha, J., and Kaminer, I. (2009), Coordinated path-following control of multiple vehicles in the presence of communication losses and time delays, SIAM J. on Control. and Optimization, vol. 48, no. 1, pp. 234-265

[7] Ghabcheloo, R., Aguiar, A., Pascoal, A., Silvestre, C., Kaminer, I. and Hespanha, J. (2006b), "Coordinated path-following control of multiple underactuated autonomous vehicles in the presence of communication failures," Proc. of the 45th IEEE CDC, San Diego, California, USA.

[8] Ghabcheloo, R., Pascoal, A., Silvestre, C. and Kaminer, I. (2006c), Group Coordination and Cooperative Control, Springer-Verlag, chapter "Coordinated Path-Following Control of Multiple Vehicles subject to Bi-directional Communication Constraints," pp. 93-111.

[9] Ghabcheloo, R., Pascoal, A., Silvestre, C. and Kaminer, I. (2007), Nonlinear coordinated path-following control of multiple wheeled robots with bi-directional communication constraints, International $\mathrm{J}$. of Adaptive Control and Signal Processing, 21(2-3), 133 - 157.

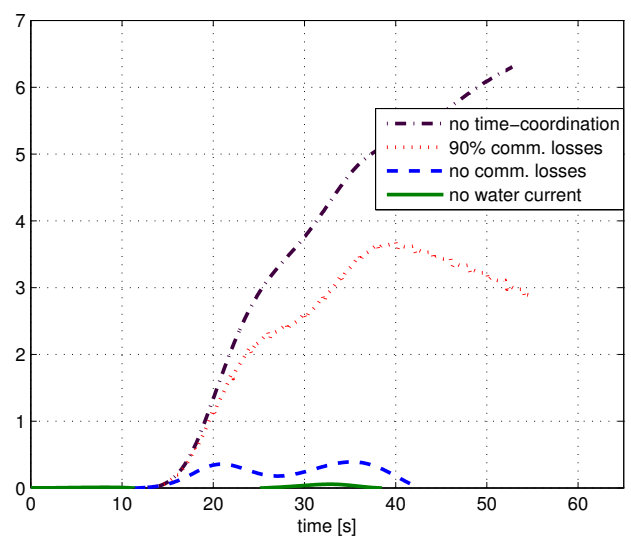

Fig. 5. Coordination error $\gamma_{1}-\gamma_{2}$

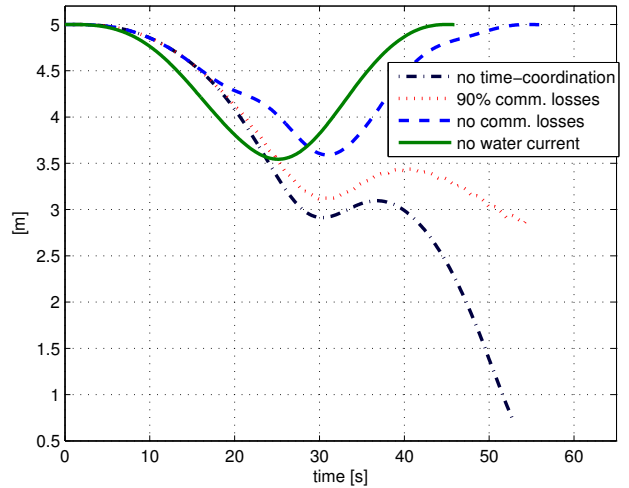

Fig. 6. Clearance $\left\|p_{1}(t)-p_{2}(t)\right\|$

[10] GREX project (2006), http : / /www(dot)grex-project(dot)eu/

[11] Jiang, Z.P., Teel, A., and Praly, L. (1994), Small gain theorem for ISS systems and applications, Math. Control, Signals and Systems., vol 7, pp. $95-120$.

[12] Kaminer, I, Yakimenko, O., Dohrokhodov, V., Pascoal, A., Hovakimyan, N., Patel, V., Cao, C., and Young A. (2007), Coordinated Path Following for Time-Critical Missions of Multiple UAVs via LI Adaptive Output Feedback Controllers, AIAA Journal of Guidance, Control, and Dynamics, Hilton Head Island, SC

[13] Lapierre, L., Soentanto, D. and Pascoal, A. (2003), "Coordinated motion control of marine robots," Proc. of the 6th IFAC Conference on Manoeuvering and Control of Marine Craft $(M C M C)$, Girona, Spain.

[14] Lin, J., Morse, A. S. and Anderson, B. D. O. (2006), The multiagent rendezvous problem - part 1: The synchronous case, SIAM J. on Control and Optimization, vol. 46, no. 6, Nov. 2007, pp. 2096-2119.

[15] Lin, Z., Francis, B. and Maggiore, M. (2007), State agreement for continuous time coupled nonlinear systems, SIAM Journal on Control and Optimization, Vol. 46, N. 1, pp 288-307.

[16] McLain, T.W. and Beard, R.W. (2000), Trajectory planning for coordinated rendezvous of unmanned air vehicles, AIAA Journal of Guidance, Control, and Dynamics, Denver, CO.

[17] Olfati-Saber, R., Fax, J.A. and Murray, R.M. (2007), Consensus and Cooperation in Networked Multi-Agent Systems, Proceedings of the IEEE Publication. Jan. 2007, vol. 95, no. 1, pp. 215-233.

[18] Shanmugavel, M. and Tsourdos, A. and Zbikowski, R. and White, B.A. and Rabbath, C.A. and Lechevin, N. (2006), "A solution to simultaneous arrival of multiple UAVs using Pythagorean hodograph curves", American Control Conference, 14-16 June 2006

[19] Siljak, D.D. (2008), Dynamic graphs, Proc. of the Int. Conf. on Nonlinear Analysis: Hybrid Systems, vol. 2, no. 2, pp. 544-567

[20] Soetanto, D., Lapierre, L. and Pascoal, A. (2003), "Adaptive, nonsingular path following, control of dynamic wheeled robots," Proc. of $I C A R$, Coimbra, Portugal.

[21] Sontag, E. D. and Wang, Y. (1996), New characterizations of input-tostate stability, IEEE Trans. on Autom. Cont., 41(9), pp. 1283-1294.

[22] Yakimenko, O. (2000), Direct method for rapid prototyping of nearoptimal aircraft trajectories,' AIAA Journal of Guidance, Control, and Dynamics, 23(5), 2000, pp. 865-875. 